\title{
Assessment of Tuberculosis Retreatment Case Rate and Its Treatment Outcomes at Adama Hospital Medical College, East Showa, Ethiopia
}

\author{
Jimma Likisa Lenjisa ${ }^{1 *}$, Birhanu Beri Tolosa ${ }^{1}$, Minyahil Alebachew Woldu ${ }^{1}$, Dumessa Edessa Negassa ${ }^{2}$ and Getu Bayisa Wakjira ${ }^{3}$ \\ ${ }^{1}$ Ambo university, College of medicine and health sciences, Department of pharmacy, Clinical Pharmacy Course and Research Team, Po box: 19 , Ambo, Ethiopia \\ ${ }^{2}$ Haramaya University, College Of Medicine and Health Sciences, Department Of Pharmacy, Clinical Pharmacy Course and Research Team, Po box: 19, Ambo, Ethiopia \\ ${ }^{3}$ Wollega University, College Of Medicine and Health Sciences, Department Of Pharmacy, Clinical Pharmacy Course and Research Team, Po box: 19, Ambo, Ethiopia
}

*Corresponding author: Jimma Likisa Lenjisa, Ambo University, College of Medicine and Health Sciences, Department of Pharmacy, Clinical Pharmacy Course and Research Team, PO box: 19, Ambo, Ethiopia, Tel: +251917305585; E-mail: jimmapharm@yahoo.com

Rec date: Oct 13, 2014, Acc date: Feb 20, 2015, Pub date: Feb 27, 2015

Copyright: (C) 2015, Lenjisa JL et al. This is an open-access article distributed under the terms of the Creative Commons Attribution License, which permits unrestricted use, distribution, and reproduction in any medium, provided the original author and source are credited.

\begin{abstract}
Background: Even though the proportion of previously treated TB patients' (retreatment cases) is increasing at alarming rate, there is scarcity of data regarding their magnitude and treatment outcomes. Therefore, this study is meant to assess TB retreatment case rate and their treatment outcomes at Adama hospital medical college (AHMC) in Ethiopia.
\end{abstract}

Methods: The study employed a retrospective cross-sectional survey where a total of 732 registered TB patients' data were reviewed between Aprils to May 2014. Data analysis was done using SPSS version 20.0.

Results: In this study, $147(20 \%)$ of 732 TB cases were registered as retreatment cases. Of these, $16(11 \%)$ relapse, $13(9 \%)$ return after defaulted (RAD), $23(16 \%)$ failure and $95(64 \%)$ were categorized as 'other' among which $61(64.9 \%)$ had sputum smear-negative pulmonary TB (PTB) and 34 (35.1\%) had extra PTB (EPTB). Retreatment case is more likely to occur in HIV positive $48(59 \%)$ individuals. Regarding their treatment outcomes, treatment success rate (cure plus treatment completed) of $65(63.7 \%)$ was obtained. Whereas $13(12.8 \%)$ registered as died, $10(9.8 \%)$ as failure, $9(8.8 \%)$ as defaulters and $5(4.9 \%)$ as transferred out cases to other health facility. HIV positive individuals are more likely $(54 \%)$ to have poor treatment outcomes.

Conclusion: The prevalence of TB retreatment cases obtained in this study was higher as compared to similar studies. Similarly, their treatment success rate (TSR) was lower as compared to the national figure. Therefore, more attention should be given to the prevention and treatment of TB retreatment cases to avoid social and economic burden of tuberculosis in Ethiopia.

Keywords: Tuberculosis; TB re-treatment case; Treatment success rate (TSR); Adama Ethiopia

\section{Introduction}

Tuberculosis (TB) is one of the most wide spread infection known in the world. Approximately 1.7 billion people or one-third of the world's population is to be infected with mycobacterium tubercle bacilli. Every year, about nine million cases of active TB disease and 2 million deaths occur globally. Most of the cases of active TB (7 million) are in Asia and Africa [1-3]. Tuberculosis is the major cause of death in developing countries; it comprises $25 \%$ of avoidable adult deaths. TB affects mostly young adults in their productive age groups because of the HIV epidemic [4-7].The annual infection rate in developing countries reached $2 \%$ or more; whereas in developed countries this figure is $0.5 \%$ [8].

Although the new tuberculosis treatment strategy based on short course chemotherapy is introduced in 1991, Africa is falling short of the world health organization's targets for case detection and treatment rates [1]. In Ethiopia, the effort of controlling tuberculosis began in the early 1960s with the establishment of TB centers and sanatorium in three major urban areas of the country.
According to the 2011 health and health related indicators of federal ministry of health (FMOH), TB is the 3rd leading cause of death in Ethiopia. During the year of 2010/11 a total of 159,017 TB cases were notified in Ethiopia. Among these 151,866 (95.5\%) were new case of $\mathrm{TB}$, all forms and 4,612 (2.9\%) were retreatment cases notified. Previously treated TB patients are classified into four categories: relapse, failure, and return after defaulted (RAD), and other. According to Ethiopian national TB guidelines, 'other' includes chronic re-treatment TB patients, recurrent smear-negative pulmonary tuberculosis (PTB) and extra-pulmonary tuberculosis (EPTB).

A global report on relapse, failure, RAD and other for Africa, southEast Asia, and western pacific regions in 2007 showed that out of 581,000 TB re-treatment cases registered $65 \%$ and $35 \%$ were registered as smear-positive and smear-negative (other) respectively $[9,10]$. These retreatment $\mathrm{TB}$ cases in turn play major role in the development of drug resistance including MDR-TB which is considered to be a global threat today. For example, according to the anti-TB drug resistance survey conducted in 2005, among 804 newly diagnosed TB cases $1.6 \%$ were found to be infected with MDR TB while from 76 previously treated TB cases, $11.8 \%$ develop MDR TB. 
Citation: Lenjisa JL, Tolosa BB, Woldu MA, Negassa DE, Wakjira GB (2015) Assessment of Tuberculosis Retreatment Case Rate and Its Treatment Outcomes at Adama Hospital Medical College, East Showa, Ethiopia . J Steroids Horm Sci 6: 1000.153.

Page 2 of 6

For the last decade (1999-2008), Ethiopia registered a total of 372,427 smear positive, $1,166,863$ new all forms and 7,582 retreatment TB cases. In its 2012, WHO reported that there was an estimated 1700 and 550 MDR TB case notified new and retreatment PTB cases in 2011 respectively in Ethiopia. Similarly, Ethiopian national drug resistance survey of 2005 estimated the prevalence of MDR-TB among new and retreatment cases was $1.6 \%$ and $12 \%$ respectively. Current evidence from the study hospital also indicated that most MDR-TB cases were emerged from retreatment cases especially among those who failed their retreatment regimens. However, there is no clear data in relation to the prevalence of TB retreatment cases, outcomes of their treatment as well as factors which affect the occurrence of retreatment and poor treatment outcomes of retreated TB cases in this hospital. Therefore, this study is designed to generate essential data on these regards and give appropriate recommendation based on the findings.

\section{Method and Material}

\section{Study area and period}

The study was conducted in AHMC, Adama town, Oromia regional state, Ethiopia. Adama town located $98 \mathrm{~km}$ east of the capital city Addis Ababa. Currently it has a total population of 311, 483 (male 154, 184 and female 157, 299).

There are different private and public health institutions in the town including one medical hospital. The information from Adama health Bureau shows that currently there are 108 health institutions in town. Of these institutions, 89 are private, 7 governmental including one medical hospital, 1 non- governmental and 11 other governmental institutions. Currently there are about 320 health professionals working in the AHMC. Of these, 10 are pharmacist, 4 druggist, 26 general practitioners, 20 specialists, 112 nurses of all type, 21 medical laboratory technicians and 5 health officers. Additionally, there are 6 wards that contain a total of 220 beds. In AHMC the diagnosis of PTB is based on clinical examination, sputum smear microscopy, and chest radiography. In most cases, diagnosis of EPTB is made based on radiography, but it was also diagnosed bacterioligically or histopathologically. According to national guideline the retreatment patients should be treated with the standard WHO category-II regimen, consisting of two months of daily streptomycin (S), rifampicin $(\mathrm{R})$, isoniazid $(\mathrm{H})$, pyrazinamide $(\mathrm{Z})$ and ethambutol $(\mathrm{E})$, followed by one month of RHZE and five months of RHE (2SRHZE/ 1RHZE/5RHE). The study was conducted from April to May, 2014.

\section{Study Design}

This study was conducted based on the retrospective cross-sectional study of retreatment TB patients (age 15 and above) using routine program data from $\mathrm{TB}$ registers and patient treatment cards registered from January, 2010 to August, 2013 at AHMC.

\section{Populations}

\section{Source population}

All the TB patients who were registered in TB clinic at AHMC for last four years from 2010 to 2013.

\section{Study Population}

All the TB patients who were registered in TB clinic at AHMC for last four years from 2010 to 2013 that fulfill the inclusion criteria.

\section{Inclusion and Exclusion Criteria}

\section{Inclusion criteria}

All registered TB patients aged 15 and above who were treated at AHMC of TB clinics from $2010-2013$.

\section{Exclusion criteria}

Poor registration or recording that could affect the analysis.

Patients that were discontinued treatment due to miss diagnosis 12 (7\%).

\section{Data Collection Method}

Data collected from registers for each patient included TB registration number, registration date, age, sex, TB classification, patient category, treatment regimens, initial sputum microscopy results and HIV status. This data was collected by TB clinic staff after permission were obtained from the concerned body. By using data collection format, then data was collected from documentary sources (secondary data).

\section{Study Variables}

\section{Dependent variables}

Prevalence TB retreatment cases

Treatment outcomes of TB retreatment cases

\section{Independent Variables}

Characteristics of patients (age, sex, pregnancy, lactation...)

Clinical characteristics of the patients such as HIV status, smear status etc....

\section{Data Analysis and Interpretation}

The collected data were entered and analyzed using Microsoft excel. Cross-tabulation was made to examine association of independent variables with outcome variables. The findings were summarized in terms of frequencies and percentages and presented using tables.

\section{Data quality control measures}

Data were cleared and checked every day for completeness and consistency.

\section{Operational definitions of terms and concept}

Smear positive pulmonary TB: A patient with two sputum specimens' positive for acid fast bacilli by microscopy.

Smear Negative pulmonary TB: A patient with three sputum specimens negative for acid fast bacilli by microscopy. 
Citation: Lenjisa JL, Tolosa BB, Woldu MA, Negassa DE, Wakjira GB (2015) Assessment of Tuberculosis Retreatment Case Rate and Its Treatment Outcomes at Adama Hospital Medical College, East Showa, Ethiopia . J Steroids Horm Sci 6: 1000.153.

Page 3 of 6

Multi drug-resistant TB: Mycobacterium tuberculosis resistant to isoniazid and rifampicin, with or without resistance to other drugs.

Extra pulmonary TB: Tuberculosis in organs other than the lungs.

Re-treatment: Treating TB patients again with failure, relapse, RAD and other.

Treatment complete: Finishing the course of treatment and/or cure.

TSR: cure plus treatment completed

\section{Ethical Consideration}

A formal letter was written from Ambo University, College of Medicine and Health Sciences, Department of pharmacy to Adama hospital medical college. Then ethical committee viewed and approved that the study used routine programmatic data that did not include any personal identifiers.

\section{Limitations and Strength of the Study}

\section{Limitation of the study}

The study was conducted only in one hospital which is may not holds true for others.

The research was not included miss diagnosed patient who discontinued treatments.
The study was not included transferred out patients to the surrounding health institutions.

The study did not include all age groups because of financial problem.

\section{Strength of the study}

The study was included all patients registered as retreatment at AHMC of TB clinics to reduce the bias formed during sampling method except age group less than 15 years because of rarely diagnosed with these age groups for retreatment $\mathrm{TB}$ and financial problem.

\section{Results}

Between January 2010 and August 2013, 732 adult TB cases were registered at AHMC. Of all TB registrations, 658 (90\%) had smear microscopy; 221 (30\%) had positive smear results. A total of 147 (20\%) were registered as retreatment cases. Compared to a new TB cases retreatment cases were more likely to be male $90(61 \%)$ versus 296 (51\%), age $15-35$ years $84(57 \%)$ versus $290(50 \%)$, and less likely to have known HIV status 65 (44\%) versus 398 (68\%). Among those with known HIV status retreatment cases were more likely to be HIV positive 48 (59\%) versus 107 (57\%) (Figure 1 and Table 1).

\begin{tabular}{|c|c|c|c|c|c|}
\hline \multirow[t]{2}{*}{ Characteristics } & \multicolumn{2}{|c|}{$\begin{array}{l}\text { New cases } \\
\text { New case }\end{array}$} & \multicolumn{2}{|c|}{$\begin{array}{l}\text { Retreatment cases } \\
\text { Retreatment case }\end{array}$} & \multirow[t]{2}{*}{$p$-value } \\
\hline & $\mathrm{N}$ & $\%$ & $\mathrm{~N}$ & $\%$ & \\
\hline \multicolumn{6}{|l|}{ Sex } \\
\hline Male & 296 & $51 \%$ & 90 & $61 \%$ & 0.1543 \\
\hline Female & 289 & $49 \%$ & 57 & $39 \%$ & \\
\hline \multicolumn{6}{|l|}{ Age } \\
\hline $15-35$ & 290 & $50 \%$ & 84 & $57 \%$ & 0.039371 \\
\hline$>35$ & 260 & $44 \%$ & 63 & $43 \%$ & \\
\hline Unknown & 35 & $6 \%$ & 0 & $0 \%$ & \\
\hline \multicolumn{6}{|l|}{ HIV status } \\
\hline Positive & 212 & $36 \%$ & 76 & $52 \%$ & 0.010889 \\
\hline Negative & 342 & $59 \%$ & 71 & $48 \%$ & \\
\hline Unknown & 31 & $5 \%$ & 0 & $0 \%$ & \\
\hline \multicolumn{6}{|l|}{ Smear status } \\
\hline Positive & 169 & $29 \%$ & 52 & $36 \%$ & 0.001536 \\
\hline Negative & 342 & $59 \%$ & 95 & $64 \%$ & \\
\hline Unknown & 74 & $12 \%$ & 0 & $0 \%$ & \\
\hline
\end{tabular}

Table 1: Distribution of patient characteristics by treatment category $(n=732)$. 
Citation: Lenjisa JL, Tolosa BB, Woldu MA, Negassa DE, Wakjira GB (2015) Assessment of Tuberculosis Retreatment Case Rate and Its Treatment Outcomes at Adama Hospital Medical College, East Showa, Ethiopia . J Steroids Horm Sci 6: 1000.153.

Page 4 of 6

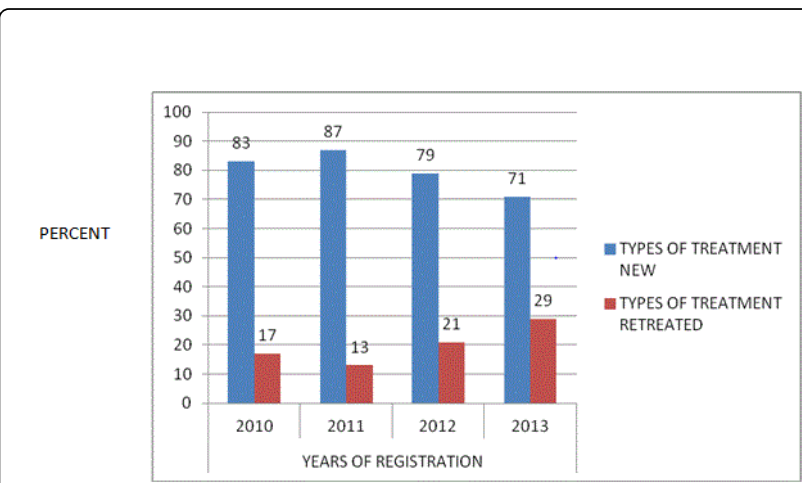

Figure 1: Trends of TB retreatment cases over four years, AHMC, Ehiopia.

\section{Treatment Outcomes of Retreatment Cases}

Treatment outcomes were recorded for $102(69 \%)$ of 147 retreatment cases. Of the 102 recorded outcomes 81 (79\%) had PTB and $21(31 \%)$ had EPTB. Treatment cards were missing for $45(31 \%)$, these patients were not included in further analysis. Of all outcomes 17 (16\%) cure rate, 48 (47\%) treatment complete, 9 (8.82\%) loss to follow up (defaulters), $13(12.75 \%)$ death, 10 (9.80\%) failure rate and 5 (4.90\%) transferred out (Figure 2 and Table 2).

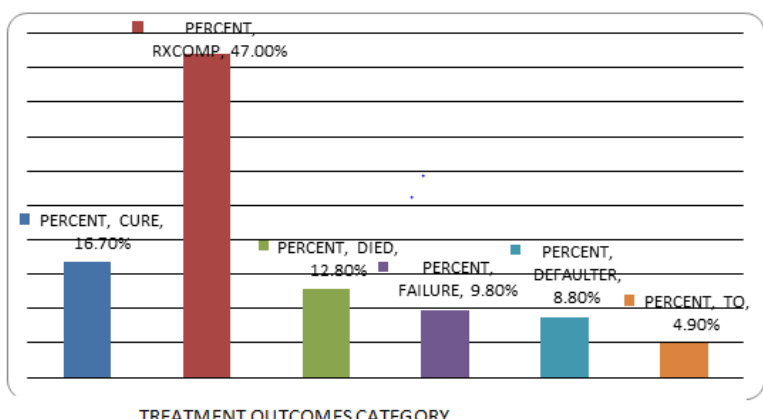

Figure 2: Treatment outcomes of TB retreatment case, AHMC, Ethiopia.

\section{Factors Associated with Treatment Outcomes of TB Retreatment}

The total TSR of the study was $65(64 \%)$ which is poor outcome compared to other studies. Results from this study indicates that females were more likely to have better treatment outcomes than males 35 (54\%) versus 30 (46\%). Treatment success was also significantly higher in smear negative 'other' than smear positive relapse, RAD and failure 47 (72\%) versus 18 (28\%) (Table 2).

\begin{tabular}{|l|l|l|l|}
\hline Characteristics & Total & Successful & $\begin{array}{l}\text { Not successful } p \text { - } \\
\text { value }\end{array}$ \\
\hline
\end{tabular}

\begin{tabular}{|c|c|c|c|c|c|}
\hline & & $\mathbf{N}$ & $\%$ & $\mathbf{N}$ & $\%$ \\
\hline \multicolumn{6}{|l|}{ Sex } \\
\hline Male & 45 & 30 & $46 \%$ & 15 & $41 \%$ \\
\hline Female & 57 & 35 & $54 \%$ & 22 & $59 \%$ \\
\hline \multicolumn{6}{|l|}{ Age } \\
\hline $15-35$ & 54 & 40 & $62 \%$ & 14 & $\begin{array}{l}38 \% \\
0.000689\end{array}$ \\
\hline$>35$ & 48 & 25 & $38 \%$ & 23 & $62 \%$ \\
\hline \multicolumn{6}{|l|}{ HIV Status } \\
\hline Positive & 64 & 44 & $68 \%$ & 20 & $\begin{array}{l}54 \% \\
0.042395\end{array}$ \\
\hline Negative & 38 & 21 & $32 \%$ & 17 & $46 \%$ \\
\hline \multicolumn{6}{|c|}{ Retreatment category } \\
\hline Relapse & 16 & 9 & $14 \%$ & 7 & $19 \%<0.001$ \\
\hline RAD & 10 & 7 & $11 \%$ & 3 & $8 \%$ \\
\hline Failure & 13 & 2 & $3 \%$ & 11 & $30 \%$ \\
\hline Other(EPTB,PTB) & 63 & 47 & $72 \%$ & 16 & $43 \%$ \\
\hline \multicolumn{6}{|l|}{ Treatment regimen } \\
\hline Category I & 48 & 27 & $56 \%$ & 21 & $44 \%<0.001$ \\
\hline Category II & 49 & 36 & $73 \%$ & 13 & $27 \%$ \\
\hline Others & 5 & 2 & $40 \%$ & 3 & $60 \%$ \\
\hline \multicolumn{6}{|l|}{ Years of Registration } \\
\hline 2010 & 29 & 23 & $79 \%$ & 6 & $21 \%<0.001$ \\
\hline 2011 & 18 & 12 & $71 \%$ & 6 & $29 \%$ \\
\hline 2012 & 25 & 16 & $64 \%$ & 9 & $36 \%$ \\
\hline 2013 & 30 & 14 & $47 \%$ & 16 & $53 \%$ \\
\hline
\end{tabular}

Table 2: Association between different factors with treatment outcomes among retreatment TB patients $(n=102)$.

\section{Discussions}

This study was done from a routine TB program setting in Adama Hospital Medical College (AHMC) to explore characteristics and treatment outcomes in previously treated adult $\mathrm{TB}$ patients registered as relapse, RAD, treatment failure, and 'other'(EPTB,PTB). In this study16 (11\%) relapse, 13 (9\%) RAD, 23 (16\%) treatment failure and 95 (64\%) 'Other' was classified as retreatment TB. Among 'other'61 (64.9\%) had smear negative PTB, 48 (51\%) were HIV positive and most were men older than 35 years.

According to Ethiopian national $\mathrm{TB}$ guidelines a retreatment regimen (2SRHZE/1RHZE/5RHE) should have been used for all retreatment cases. But more than half of patients classified as 'other' and treatment failure were inappropriately treated with category I regimen $(2 \mathrm{RHZE} / 4 \mathrm{RH})$ and others category regimen like (2SHE/ 
Citation: Lenjisa JL, Tolosa BB, Woldu MA, Negassa DE, Wakjira GB (2015) Assessment of Tuberculosis Retreatment Case Rate and Its Treatment Outcomes at Adama Hospital Medical College, East Showa, Ethiopia . J Steroids Horm Sci 6: 1000.153.

Page 5 of 6

10HE). However, Overall TSR was low among retreatment TB patients receiving category II regimen suggesting that; this regimen may also not be the most appropriate. Because as WHO standardization, TSR for high burden TB countries like Ethiopia should be $85 \%$. Therefore, this study indicates $65(64 \%)$ TSR which was poor results compared to WHO standardization.

There are several possible explanation for larger number of 'other' smear negative $\mathrm{TB}$ and were HIV positive, raising question about whether these were truly recurrent smear negative PTB and/or EPTB as result of re-infection or reactivation or whether they had HIV related disease that was miss diagnosed as TB. It is also possible that other pulmonary or cardiac disease that was miss diagnosed as TB. As a result, miss diagnosis is a great problem in AHMC next to patient non-adherence to anti-TB drugs. Adoption of new accurate diagnostic tool such as GeneXpert MTB/RIF, culture test and drug sensitivity test (DST) might help to reduce miss diagnosis in this group of patients which is currently functioning only at regional laboratory.

According to drug administration and control authority of Ethiopian standard treatment guideline WHO 2010 recommendation previously treated TB patients should be undergone DOT system both at initiation and continuation phases. But at AHMC TB clinic retreatment TB patients were served similar to new TB patients DOT system for only 2 months.

Comparing to other studies like in Israel, Addis Ababa and Gambella regional hospital TB retreatment cases were larger 147 (20\%) in AHMC; I also observed different TSR among retreatment cases. In my study the TSR result is better than similar study done in Israel and Addis Ababa. But according to the study conducted in Gambella regional hospital my TSR result was low. The most important reason for low TSRs were loss to follow up $9(8.82 \%)$, treatment failure 10 (9.80\%) and death $13(12.75 \%)$. This proportion indicates a need closer follow up of these groups during TB treatment, particularly as loss to follow up may include hidden deaths. As this study indicates the number of previously treated patients were increasing from the year of registration data recorded evidence based documents. This also, shows there is a poor care for retreatment patients from health care TB staffs at Adama hospital medical college.

Also, despite the overall low treatment outcome rates cure rate for smear positive relapse significantly higher than cure rate in smear negative other $7 \mathrm{vs} 3$ (41\% versus $18 \%$ ). There are various possible explanations: relapse patients have microbiologically proven $\mathrm{TB}$ in contrast to 'other' cases, which as discussed above may be mixed of true TB, HIV related disease and other pulmonary or cardiac disease. Given high HIV infection rates in 'other' cases and the inclusion of condition that may not respond to TB treatment, only moderate proportion might improve clinically.

Generally, the retreatment group had high retreatment failure rates $10(9.80 \%)$ outcome s which may indicates a high prevalence of MDR$\mathrm{TB}$, so this does requires more in depth study. The reason of increasing failure rate to result MDR-TB may be non-adherence of patients, in availability of drugs, less care from TB staff HW, false laboratory result, drug resistance, and etc. My result showed that males had lower TSR and were more likely to default or die while on TB treatment, and this may reflect low compliance with $\mathrm{TB}$ treatment therapy.

In my study the following limitations viewed. First, 45 (31\%) of treatment cards for retreatment patients were lost. This introduces bias if patients without treatment cards had different outcomes than those with treatment cards. A considerable proportion of adverse effects were recorded on treatment card, but not on TB register. Second, 12 (7\%) miss diagnosed patients that discontinued TB treatment regimen also disturb the quality of this study.

Despite these limitations, the study findings are useful inform policy and programs in Ethiopia and other comparable setting to improve care TB patients.

\section{Conclusion and Recommendation}

\section{Conclusion}

This study shows that the treatment failure and 'other' TB category constitutes a sizeable population of retreatment patients and is poorly managed. In AHMC giving up dated regular information to health provider particularly physicians and laboratory technicians may reduce poor management and misdiagnosis of retreatment TB. The high proportion of missing treatment cards suggest that less priority is given to retreatment patients. The treatment success may be improved by adoption of more accurate diagnostic tools such as GeneXpert MTB/RIF and sufficient training and regular supervision of TB staff.

Generally, in management of previously treated TB patients following a routine programmatic that was settled by Ethiopian, FMOH to control TB, TB/HIV and leprosy plan of the 2013 is a good practice to bring high TSR.

\section{Recommendation}

Strengthening laboratory of referral hospitals to the capacity of performing GeneXpert MTB/RIF, culture and DST.

Separating TB treatment card room solely from whole patient card room.

Implementation of DOTs plus strategy regardless of age group both at initiation and continuation phases.

Encouraging operational research towards evidence based diagnosis of TB patients.

Giving sufficient training and regular supervision to TB staffs.

\section{Competing Interests}

The authors declare that they have no competing interests.

\section{Authors Contributions}

JLL and BBT had involved in the design, implementation of the study, performed statistical analysis, and drafted the manuscript. MAW, DEN and GBW conceived and designed the study, involved in the implementation of the study, performed statistical analysis and critically revised the manuscript. All the authors read and approved the final manuscript.

\section{Acknowledgement}

We would like to extend our gratitude to Ambo university department of pharmacy for whole rounded support. Furthermore, we would like to express our warmest gratitude to all Tuberculosis (TB) clinic staff and others for their continuous support. We would also like to thank East showa zone health Bureau and Adama hospital medical 
Citation: Lenjisa JL, Tolosa BB, Woldu MA, Negassa DE, Wakjira GB (2015) Assessment of Tuberculosis Retreatment Case Rate and Its Treatment Outcomes at Adama Hospital Medical College, East Showa, Ethiopia . J Steroids Horm Sci 6: 1000.153.

doi:10.4172/2157-7536.1000.153

Page 6 of 6

college administrate for their help during data gathering process for this thesis.

\section{References}

1. World Health Organization Report (2011) Global Tuberculosis Control. WHO/HTM/TB/2011; 16

2. World Health Organization (2012) Global tuberculosis control; WHO report 2012.

3. World Health Organization Report (2013) Global Tuberculosis Control. WHO/HTM/TB/2013.11.

4. Kaufmann SH, Parida SK. (2008) Tuberculosis in Africa: learning from pathogenesis for biomarker identification. Cell Host Microbe; 4: 219-28.

5. Ministry of Health of Ethiopia. (2011) First Ethiopia National Population based Tuberculosis survey Tuberculosis. Addis Ababa: MOH.

6. FMOH. (2013) Guidelines for clinical and programmatic management of TB, TB/HIV and leprosy. Addis Ababa, Ethiopia. (5th edition).
7. Daniel MN, Wondemagegn ML, Bayeh AB, et al. (2014) Prevalence of Multi Drug Resistant Tuberculosis among Presumptive Multi Drug Resistant Tuberculosis Cases in Amhara National Regional State, Ethiopia. J Mycobac Dis, 4:3.

8. Michael KW, Belachew T, Jira C. (2004) Tuberculosis defaulters from the "dots" regimen in Jimma zone, southwest Ethiopia. Ethiop Med J Oct; 42: 247-53.

9. Fantahun B, Berhanu A, Tewodros D, Belay A, Woghata T, et al. (2013) A retrospective study on the outcomes of tuberculosis treatment in Felege Hiwot Referral Hospital, Northwest Ethiopia. International Journal of Medicine and Medical Sciences.; 5: 85-91.

10. WHO (2011) towards universal access to diagnosis and treatment of multidrug-resistant and extensively drug-resistant tuberculosis by 2015: WHO progress report. 